\title{
Characterization of miniaturized metamaterial resonators coupled to planar transmission lines through parameter extraction
}

Cite as: J. Appl. Phys. 104, 114501 (2008); https://doi.org/10.1063/1.3021109

Submitted: 16 July 2008 . Accepted: 01 October 2008 . Published Online: 01 December 2008

F. Aznar, M. Gil, J. Bonache, L. Jelinek, J. D. Baena, R. Marqués, and F. Martín

\section{ARTICLES YOU MAY BE INTERESTED IN}

Split ring resonator-based left-handed coplanar waveguide

Applied Physics Letters 83, 4652 (2003); https://doi.org/10.1063/1.1631392

Electric-field-coupled resonators for negative permittivity metamaterials

Applied Physics Letters 88, 041109 (2006); https://doi.org/10.1063/1.2166681

Complex permittivity measurement using metamaterial split ring resonators Journal of Applied Physics 121, 054101 (2017); https://doi.org/10.1063/1.4975111

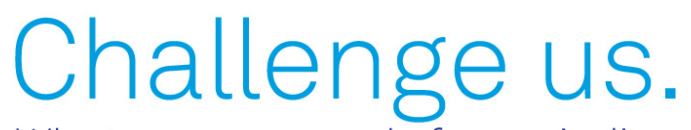

What are your needs for periodic signal detection?

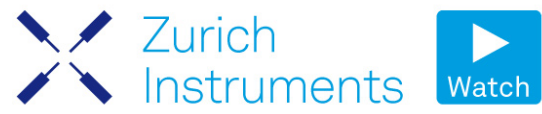

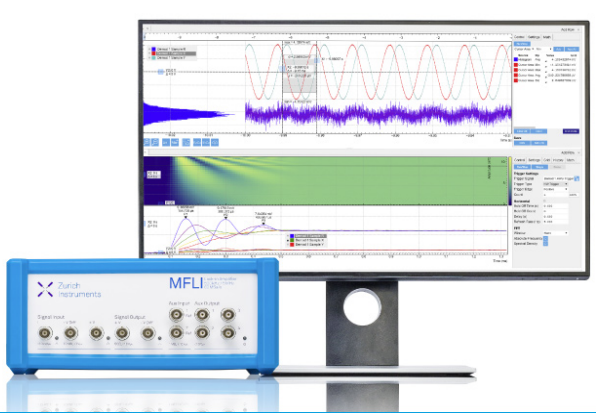




\title{
Characterization of miniaturized metamaterial resonators coupled to planar transmission lines through parameter extraction
}

\author{
F. Aznar, ${ }^{1, a)}$ M. Gil, ${ }^{1}$ J. Bonache, ${ }^{1}$ L. Jelinek, ${ }^{2}$ J. D. Baena, ${ }^{3}$ R. Marqués, ${ }^{2}$ and \\ F. Martín ${ }^{1, b)}$ \\ ${ }^{1}$ Departament d'Enginyeria Electrònica, GEMMA/CIMITEC, Universitat Autònoma de Barcelona, 08193 \\ Bellaterra (Barcelona), Spain \\ ${ }^{2}$ Departamento de Electrónica y Electromagnetismo, Universidad de Sevilla, Avda. Reina Mercedes, s/n, \\ 41012 Seville, Spain \\ ${ }^{3}$ Departamento de Física, Universidad Nacional de Colombia, Carrera 30 N45-30 Bogotá D.C., Colombia
}

(Received 16 July 2008; accepted 1 October 2008; published online 1 December 2008)

\begin{abstract}
In this paper, a method for obtaining the electrical characteristics of metamaterial resonators coupled to planar transmission lines is proposed. This parameter extraction technique is based on the comparison between the measured (or full wave electromagnetic simulated) transmission and reflection characteristics of a host line loaded with such resonators and those obtained from its lumped element equivalent circuit model (previously reported by some of the authors). The resonant particles considered in this study are split ring resonators, spiral resonators, and other electrically small resonant particles based on two metal levels. The interest in this technique lies in the lack of analytical models providing the electrical parameters of several of the considered ultrasmall resonator topologies (due to their complexity). From the extracted parameters, it is concluded that the circuit models predict very accurately the frequency responses of the considered structures for the different resonators under study. There is an increasing interest in the synthesis of metamaterial transmission lines with extremely small unit cell size. In order to achieve dimensions close to (or even below) $\lambda_{g} / 100$, it is imperative to use complex resonator topologies that combine broadside coupling and extremely elongated metallic strips (etched in different metal levels connected through vias). This justifies the proposed technique. Thus, this work is useful in aiding the synthesis of microwave components based on resonant type metamaterial transmission lines. (C) 2008 American Institute of Physics. [DOI: 10.1063/1.3021109]
\end{abstract}

\section{INTRODUCTION}

Resonant type metamaterial transmission lines were first proposed in 2003 by Martín et al. ${ }^{1}$ by periodic loading of a coplanar waveguide (CPW) structure with split ring resonators (SRRs) (Ref. 2) and shunt connected strips. In such structures, the metallic strips provide a negative value of the effective dielectric permittivity $\varepsilon_{\text {eff }}$ below a certain frequency (plasma frequency $f_{p}$ ), whereas the SRRs are responsible for the negative effective permeability $\mu_{\text {eff }}$ in a narrow band above their resonance frequency $f_{o}$. By designing the structure with $f_{o}<f_{p}, \varepsilon_{\text {eff }}$ and $\mu_{\text {eff }}$ are simultaneously negative in a certain frequency band, and left handed (or backward) wave propagation in that region occurs. Alternatively, left handed transmission lines can be implemented by loading a host line with complementary split ring resonators (CSRRs) and series (capacitive) gaps. ${ }^{3,4}$ In this case, the negative effective permittivity and permeability are provided by the CSRRs and the gaps, respectively. The physics and applications of these resonant type metamaterial transmission lines are exhaustively discussed in the book coauthored by two of the authors ${ }^{5}$ (other books that focus on the CL-loaded approach of metamaterial transmission lines have also been recently published $^{6,7}$ ).

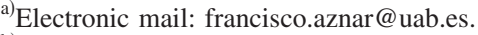

b) Tel.: $3493 \quad 58135$ 22. FAX: $3493 \quad 58126$ 00. Electronic mail: ferran.martin@uab.es.
}

There are two main aspects of metamaterial transmission lines that make them attractive for $\mathrm{RF} /$ microwave circuit design: (i) their small dimensions and (ii) the possibility of tailoring (to some extent) the dispersion diagram of such lines. The small dimensions are due to the electrically small size of the metamaterial resonators (SRRs and CSRRs, among others), which is in turn due to the high coupling between the individual rings forming the particles ${ }^{8,9}$ (or to self-coupling in certain resonators based on spiral topologies). The dispersion diagram of metamaterial transmission lines can be controlled by virtue of the higher number of degrees of freedom of these resonant type metamaterial transmission lines. This makes it possible to achieve functionalities not realizable with conventional lines, such as the synthesis of multiband components ${ }^{10}$ or components with enhanced operative bandwidths. ${ }^{11-14}$

Most resonant type metamaterial transmission lines and applications reported so far have been implemented by means of CSRRs etched either in the ground plane ${ }^{5}$ or in the conductor strip $^{15}$ of microstrip transmission lines. These lines have been exhaustively studied in the literature. ${ }^{16-18}$ With regard to SRR-loaded lines, a circuit model describing such lines has been previously reported. The validation of this model has been done by comparing experimental data obtained from fabricated prototypes with circuit simulations, ${ }^{1}$ where the circuit parameters have been inferred from models valid under very restrictive conditions ${ }^{19}$ (i.e., 
such circuits elements have not been derived from parameter extraction). This explains certain discrepancies between the results of simulation and experiment in previous works. ${ }^{1}$ In this work, a method for extracting the circuit parameters of metamaterial transmission lines loaded with SRRs or with other resonators magnetically coupled to the line is proposed. As will be shown, the circuit models with extracted parameters accurately describe the behavior of these metamaterial transmission lines, regardless of the resonator type and host line configuration (microstrip, CPW, etc.).

One relevant aspect of magnetically coupled metamaterial resonators is the possibility of significantly reducing their size by using two metal levels or the combination of two metal levels and metallic vias. For instance, broadside coupled split ring resonators (BC-SRRs), where two open rings are etched on opposite sides (face to face) of a dielectric slab, can be made electrically very small provided the thickness of the dielectric is also very small. ${ }^{19}$ There has also been report of the notable size reduction in broadside coupled spiral resonators (BC-SRs) ${ }^{20}$ where the spirals are implemented by etching different metal loops at both sides of a dielectric layer connected through metallic vias. For certain resonator types, the complexity is so important that the analytical determination of the effective inductance and capacitance is not possible. Moreover, in simpler resonators (such as SRRs) analytical models do exist, but they are valid under very restrictive conditions, that is, without the presence of the host line. Thus, the parameter extraction technique proposed in this work is of interest since it directly provides the circuit elements of those circuits modeling the resonatorloaded lines, this being of interest for metamaterial and microwave circuit design. It will be also shown that the parameter extraction is coherent with the parameters of the isolated particles. Obviously, this will be done on the basis of transmission lines loaded with resonators whose electrical characteristics can be analytically inferred. This will further strengthen the validity of the proposed models and the parameter extraction technique.

\section{THE MODEL AND PARAMETER EXTRACTION}

The former model describing the unit cell of metamaterial transmission lines loaded with magnetically coupled resonators (SRRs, SRs, etc.) and shunt connected metallic elements is depicted in Fig. 1(a). ${ }^{1}$ In Figs. 1(b) and 1(c) the layouts of typical unit cells corresponding to left handed CPW and microstrip lines, respectively, are depicted. In the CPW configuration, the SRRs are paired in the lower substrate side (where they are etched), beneath the slots of the structure and centered with the shunt strips. The negative effective permittivity is achieved by means of the shunt connected strips. In microstrip lines, the SRRs are etched in pairs on the upper substrate side, adjacent to the conductor strip. In this case, the metallic vias are responsible for the negative permittivity of the structure. As pointed out in Ref. 1 , the validity of the lumped element model [Fig. 1(a)] in describing the structures in Figs. 1(b) and 1(c) is subject to the small electrical size of the unit cells. In addition to this, the coupling between adjacent resonators and losses is ne-

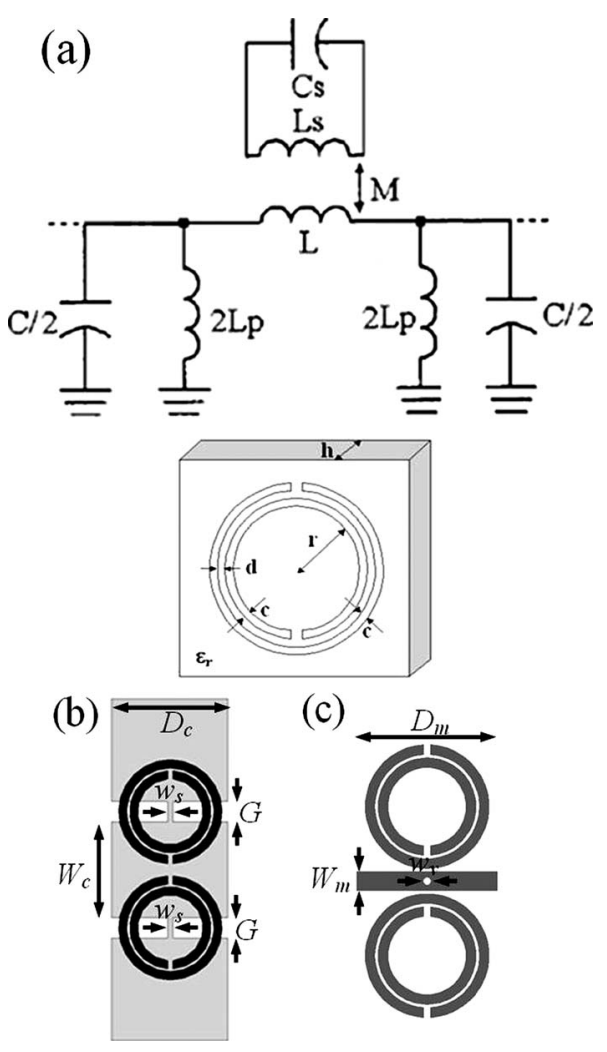

FIG. 1. Lumped element equivalent circuit for the basic left handed cell (a). Layouts of the considered CPW (b) and microstrip (c) structures combining SRRs with shunt strips and one via, respectively.

glected. It has been found that the coupling between adjacent resonant elements is significant only in square or rectangular tiny spaced resonators. ${ }^{21}$ [This coupling is the origin of magnetoinductive waves in chains of SRRs (Refs. 22-25) or electroinductive waves in CSRR arrays. ${ }^{26}$ ] In reference to Fig. 1(a), $L$ and $C$ are the per-section inductance and capacitance of the line, $L_{p}$ models the inductance of the vias (microstrip structure) or metallic strips (CPW configuration), the magnetically coupled resonators are described by means of the resonant tank constituted by the inductance $L_{s}$ and the capacitance $C_{s}$, and, finally, their magnetic coupling to the line is described by the mutual inductance $M$. As compared to the circuit model in Ref. 1, the model in Fig. 1(a) describes the whole unit cell of the artificial lines. That is, we have not applied now the magnetic wall concept, in spite of the symmetry of the structure (in reference to a central plane oriented along the line and orthogonal to it) and excitation. The reason is that in order to extract the parameters, comparison to either simulation (electromagnetic) or experiment is required (as will be discussed later), and it involves the whole structure. Following a similar analysis to that reported in Refs. 1 and 27, the circuit in Fig. 1(a) can be transformed to the circuit model depicted in Fig. 2, where

$$
\begin{aligned}
L_{s}^{\prime} & =2 M^{2} C_{s} \omega_{o}^{2}, \\
C_{s}^{\prime} & =\frac{L_{s}}{2 M^{2} \omega_{o}^{2}}, \\
L^{\prime} & =L-L_{s}^{\prime},
\end{aligned}
$$




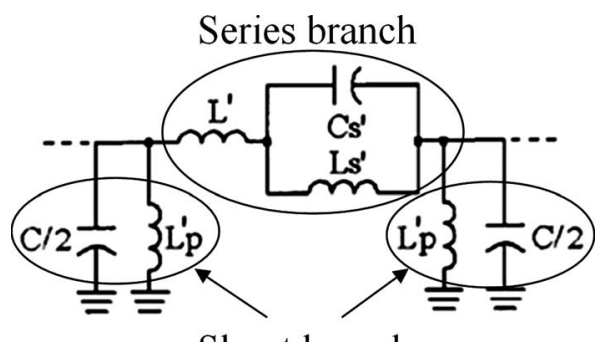

Shunt branch

FIG. 2. Transformed $\pi$-circuit model of the basic left handed cell.

$$
L_{p}^{\prime}=2 L_{p}
$$

However, it has been recently demonstrated by the authors that in order to accurately describe the frequency response of left handed lines loaded with magnetically coupled resonators through a lumped element model, it is necessary to locate the inductance of the shunt strips (or vias) as Fig. 3 illustrates. ${ }^{28}$ This circuit can also be transformed into that in Fig. 2. However, the transformation equations are no longer those given by expressions (1)-(4) but by ${ }^{28}$

$$
\begin{aligned}
& L_{s}^{\prime}=2 M^{2} C_{s} \omega_{o}^{2} \frac{\left(1+\frac{L}{4 L_{p}}\right)^{2}}{1+\frac{M^{2}}{2 L_{p} L_{s}},} \\
& C_{s}^{\prime}=\frac{L_{s}}{2 M^{2} \omega_{o}^{2}}\left(\frac{1+\frac{M^{2}}{2 L_{p} L_{s}}}{1+\frac{L}{4 L_{p}}}\right)^{2}, \\
& L^{\prime}=\left(2+\frac{L}{2 L_{p}}\right) \frac{L}{2}-L_{s}^{\prime}, \\
& L_{p}^{\prime}=2 L_{p}+\frac{L}{2} .
\end{aligned}
$$

Notice, however, that in the absence of the shunt connected strips or vias, $L_{p} \rightarrow \infty$ and expressions (5)-(8) coincide with expressions (1)-(4). As explained in Ref. 28, the model in Fig. 3 is an improved equivalent circuit of SRR-loaded left

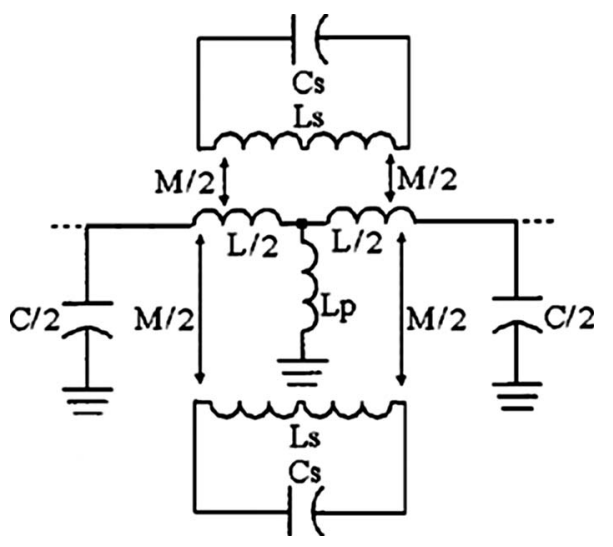

FIG. 3. Circuit model modified to describe more accurately the physical behavior of the left handed cells. handed lines, where the element parameters have the physical interpretation given before. [Notice that $L_{p}$ cannot be linked to the shunt strips or vias in the model in Fig. 1(a).] However, this model also transforms to the $\pi$-circuit in Fig. 2 [with transformation equations (5)-(8)]. The elements of the $\pi$-circuit depicted in Fig. 2, which models the unit cell of resonant type left handed transmission lines loaded with magnetically coupled resonators, can be inferred from the measured or simulated reflection and transmission coefficients of such unit cells according to the method described below.

\section{A. The parameter extraction technique}

Since the number of parameters of the circuit model in Fig. 2 is five, we also need five conditions to univocally determine such parameters. From the representation of the reflection coefficient of a single unit cell, $S_{11}$, in the Smith chart, two conditions are obtained. On one hand, we can determine the frequency that nulls the series reactance, $f_{s}$, from the intercept of $S_{11}$ with the unit conductance circle. This is obvious since at this frequency, the real part of the admittance seen from the ports is simply the admittance of the opposite port, that is, $Y_{o}=\left(Z_{o}\right)^{-1}=(50 \Omega)^{-1}=0.02 \mathrm{~S}$. Hence, $S_{11}$ must be allocated in the unit conductance circle at $f_{s}$, as illustrated in the example provided in Sec. II B. This frequency is given by the following expression:

$$
f_{s}=\frac{1}{2 \pi} \sqrt{\frac{1}{L_{s}^{\prime} C_{s}^{\prime}}+\frac{1}{L^{\prime} C_{s}^{\prime}}} .
$$

On the other hand, the susceptance of the unit cell seen from the ports at $f_{s}$, which can be inferred from the Smith chart, is

$$
B\left(\omega_{s}\right)=\frac{C L_{p}^{\prime} \omega_{s}^{2}-2}{L_{p}^{\prime} \omega_{s}},
$$

with $\omega_{s}=2 \pi f_{s}$. Another condition concerns the parallel resonator of the series branch. Namely, the resonance frequency of this resonator is given by

$$
f_{z}=\frac{1}{2 \pi} \sqrt{\frac{1}{L_{s}^{\prime} C_{s}^{\prime}}} .
$$

Notice that this frequency does not coincide with the intrinsic resonance frequency of the magnetically driven resonator, $f_{o}$ (which is the resonance frequency of the tank formed by $L_{s}$ and $C_{s}$ ). The frequency $f_{z}$ [expression (11)] can be easily obtained from the transmission coefficient $S_{21}$ of the unit cell since at this frequency the series branch is opened and the whole power injected from the input port is reflected back to the source. Thus, the transmission coefficient nulls (transmission zero frequency) and $f_{z}$ can be easily identified from the representation of the transmission coefficient in a decibel scale.

Another condition can be deduced from the phase of the transmission coefficient, $\phi_{S 21}$. At the frequency where $\phi_{S 21}$ $=90^{\circ}, f_{\pi / 2}$, the electrical length of the unit cell, $\phi=\beta l(\beta$ being the phase constant and $l$ the length of the unit cell), is 
$\phi\left(f_{\pi / 2}\right)=-90^{\circ} .{ }^{17}$ Since the dispersion relation of a periodic structure consisting of cascaded unit cells, as those in Fig. 2, is given by

$$
\cos \phi=1+\frac{Z_{s}(\omega)}{Z_{p}(\omega)},
$$

with $Z_{s}$ and $Z_{p}$ being the series and shunt impedances, respectively, of the $\pi$-circuit model, it follows that

$$
Z_{s}\left(\omega_{\pi / 2}\right)=-Z_{p}\left(\omega_{\pi / 2}\right),
$$

with $\omega_{\pi / 2}=2 \pi f_{\pi / 2}$. Expressions (9)-(11) and (13) are four of the five conditions needed to univocally determine the circuit parameters in Fig. 2. Indeed, by removing the shunt connected vias or strips in the layouts in Fig. 1, we can represent the corresponding reflection coefficient on a Smith chart and obtain the susceptance seen from the ports at that frequency where $S_{11}$ intercepts the unit conductance circle. Since this is simply the susceptance of the line capacitance (provided $L_{p}$ has been removed), we can thus univocally determine $C$. Hence, this is the fifth condition that is required to extract the circuit parameters of the circuit model in Fig. 2.

\section{B. Validation of the model and results}

The presented method has been applied in extracting the circuit model parameters (Fig. 2) of different structures. In this section, we will illustrate the validity of both, the model and the parameter extraction method, by means of the application of the technique to a microstrip line and to a CPW left handed structure. The microstrip line is loaded with a pair of SRRs and a shunt connected via, ${ }^{27}$ as depicted in Fig. 1(c). The dimensions of the SRRs are (in reference to Fig. 1) $c$ $=0.6 \mathrm{~mm}, d=0.2 \mathrm{~mm}$, and $r=2.4 \mathrm{~mm}$; the diameter of the via is $w_{v}=0.4 \mathrm{~mm}$; and the conductor strip width is $W_{m}$ $=1.15 \mathrm{~mm}$ and the length $D_{m}=8.6 \mathrm{~mm}$. The considered substrate is Rogers RO3010 with thickness $h=1.27 \mathrm{~mm}$ and dielectric constant $\varepsilon_{r}=10.2$. The reflection coefficient of the structure (obtained from full wave electromagnetic simulation by means of the AGILENT MOMENTUM commercial software) is depicted on a Smith chart in Fig. 4(a) and both the reflection and transmission coefficients are simultaneously depicted in a decibel scale in Fig. 4(b). The phase of the transmission coefficient (also obtained from full wave electromagnetic simulation) is also depicted in Fig. 4(b). We have applied the parameter extraction technique to this structure (the results are given in Table I). From the extracted parameters, we have obtained the frequency response (reflection and transmission coefficients and phase response) from the electrical simulation (using the AGILENT ADS software) of the circuit model. The results of these circuit simulations are also depicted in Fig. 4(b) to be easily compared with the results obtained from the electromagnetic simulation of the layout. As can be appreciated, the circuit model describes the behavior of the structure in Fig. 1(c) with excellent accuracy [the circuit and electromagnetic simulations in Fig. 4(b) are roughly undistinguishable]. This points out the validity of the model and the ability of the reported technique to provide the circuit parameters of the structure.
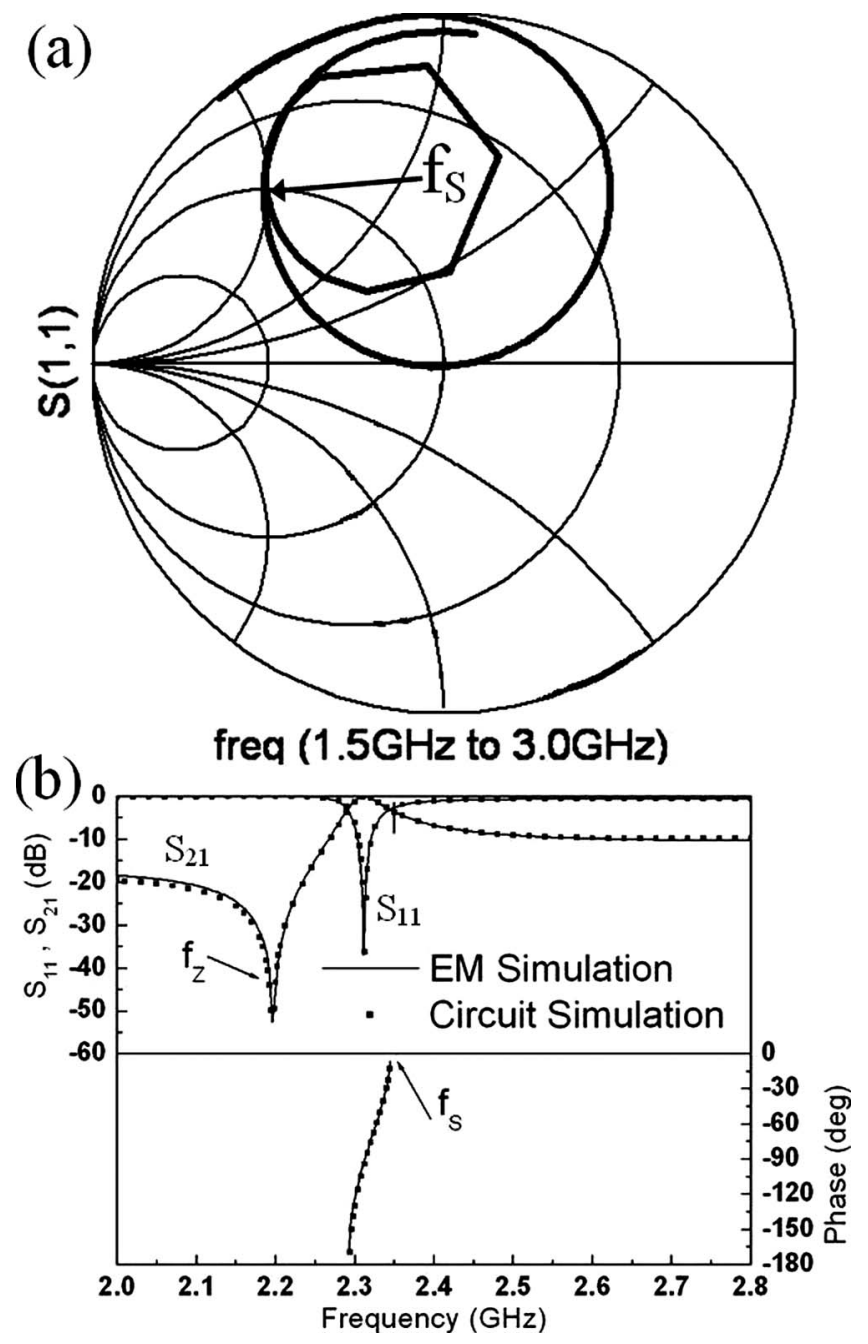

FIG. 4. Reflection coefficient on the Smith chart (a); frequency response (reflection, $S_{11}$, and transmission, $S_{21}$, coefficients) depicted in a decibel scale and the dispersion relation (b) for a left handed cell based on a microstrip structure. The considered substrate is Rogers RO3010 with thickness $h=1.27 \mathrm{~mm}$ and dielectric constant $\varepsilon_{r}=10.2$. Relevant dimensions are ring width $c=0.6 \mathrm{~mm}$; distance between the rings, $d=0.2 \mathrm{~mm}$; and internal radius $r=2.4 \mathrm{~mm}$. For the microstrip structure the strip line width is $W_{m}$ $=7 \mathrm{~mm}$ and the length is $D_{m}=8.6 \mathrm{~mm}$; the diameter of via is $w_{v}$ $=0.4 \mathrm{~mm}$. The results of the circuit simulation with extracted parameters are depicted using symbols.

The same parameter extraction method has been used for the CPW transmission line loaded with a pair of SRRs and two signal-to-ground strips. ${ }^{1}$ The SRRs are located beneath the slots of the CPW structure with the slits aligned with the

TABLE I. Extracted parameters for the circuit shown in Fig. 2 for microstrip and CPW structures with and without shunt metallic elements.

\begin{tabular}{lccccc}
\hline \hline Structure & $\begin{array}{c}C \\
\mathrm{pF})\end{array}$ & $\begin{array}{c}L^{\prime} \\
(\mathrm{nH})\end{array}$ & $\begin{array}{c}L_{p}^{\prime} \\
(\mathrm{nH})\end{array}$ & $\begin{array}{c}C_{s}^{\prime} \\
(\mathrm{pF})\end{array}$ & $\begin{array}{c}L_{s}^{\prime} \\
(\mathrm{nH})\end{array}$ \\
\hline \multicolumn{7}{c}{ Including the shunt } \\
Microstrip & 1.72 & 11.86 & 2.04 & 3.16 & 1.66 \\
CPW & 2.35 & 4.32 & 1.81 & 4.42 & 1.45 \\
\multicolumn{7}{c}{} \\
Microstrip & 1.72 & 3.02 & $\ldots$ & 45.11 & 0.11 \\
CPW & 2.35 & 2.06 & $\cdots$ & 20.29 & 0.27 \\
\hline \hline
\end{tabular}




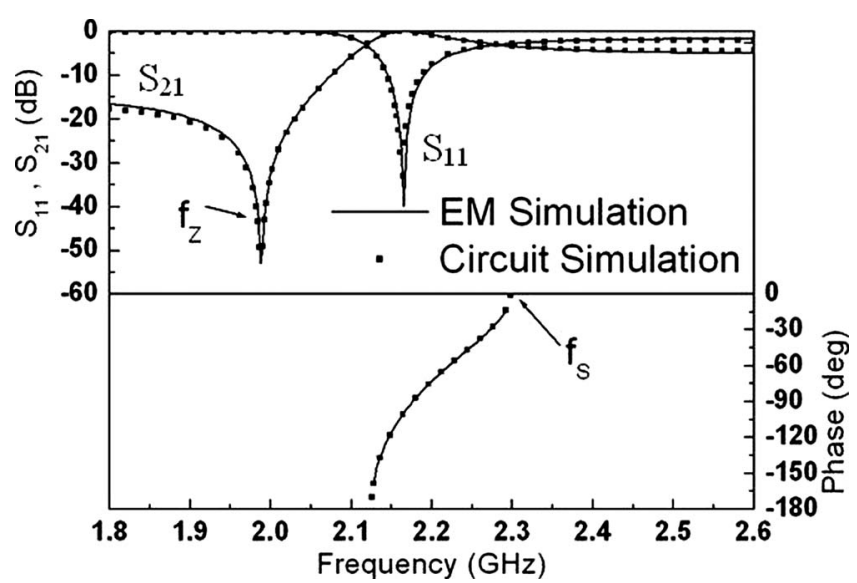

FIG. 5. Frequency response (reflection, $S_{11}$, and transmission, $S_{21}$, coefficients) depicted in a decibel scale and the dispersion relation for a left handed cell based on a CPW structure. The considered substrate is Rogers RO3010 with thickness $h=1.27 \mathrm{~mm}$ and dielectric constant $\varepsilon_{r}=10.2$. Relevant dimensions are ring width $c=0.6 \mathrm{~mm}$; distance between the rings, $d$ $=0.2 \mathrm{~mm}$; and internal radius $r=2.4 \mathrm{~mm}$. For the $\mathrm{CPW}$ structure the central strip width is $W_{c}=7 \mathrm{~mm}$, the width of the slots is $G=1.48 \mathrm{~mm}$, and the length is $D_{c}=8.6 \mathrm{~mm}$; the shunt strip width is $w_{s}=0.4 \mathrm{~mm}$. The results of the electrical simulation with extracted parameters are depicted using symbols.

shunt connected strips. In reference to Fig. 1(b), the SRR dimensions are $c=0.6 \mathrm{~mm}, d=0.2 \mathrm{~mm}$, and $r=2.4 \mathrm{~mm}$; the slot and central strip widths of the host CPW are $G$ $=1.48 \mathrm{~mm}$ and $W_{c}=7 \mathrm{~mm}$, respectively, with a length $D_{c}$ $=8.6 \mathrm{~mm}$ and the shunt metallic strip width is $w_{s}=0.4 \mathrm{~mm}$. The relative permittivity of the considered dielectric is $\varepsilon_{r}$ $=10.2$ with thickness $h=1.27 \mathrm{~mm}$ (Rogers RO3010). The electrical (from the extracted parameters given in Table I) and electromagnetic simulations of the structure are depicted in Fig. 5. Again, the agreement between circuit and electromagnetic simulations is excellent.

To further demonstrate this parameter extraction method, it has also been applied for the two previous structures after removing the shunt strips and the via in the CPW and the microstrip structure, respectively. In these cases, the electrical circuit has four elements (Fig. 2 excluding $L_{p}^{\prime}$ ). The extracted parameters can be seen in Table I, whereas the frequency responses are presented in Fig. 6 with the phase for these two structures. Comparing the frequency responses for the left handed structures in Figs. 4(b) and 5 with the negative-permeability structures in Figs. 6(a) and 6(b), the conservation of the zero-degree phase frequency $f_{s}$ and a shift in the transmission zero frequency $f_{z}$ can be observed. ${ }^{28}$

In order to generalize this model and its parameter extraction technique, a CPW left handed cell has been loaded with some different subwavelength resonators. ${ }^{20,29}$ The resonators that have been considered and their lengths in terms of the guided wavelength $\left(\lambda_{g}\right)$ are broadside coupled nonbianisotropic split ring resonator (BC-NB-SRR) $\left(\lambda_{g} / 29\right)$, broadside coupled spiral resonator with two turns [BC$\operatorname{SR}(2)]\left(\lambda_{g} / 49\right)$, and broadside coupled spiral resonator with four turns $[\mathrm{BC}-\mathrm{SR}(4)]\left(\lambda_{g} / 117\right)$. Figure 7 shows the topologies of these three resonators, the layout of the three metal layer configuration of a CPW loaded with a pair of metamaterial resonators and shunt strips, and the frequency response (a)
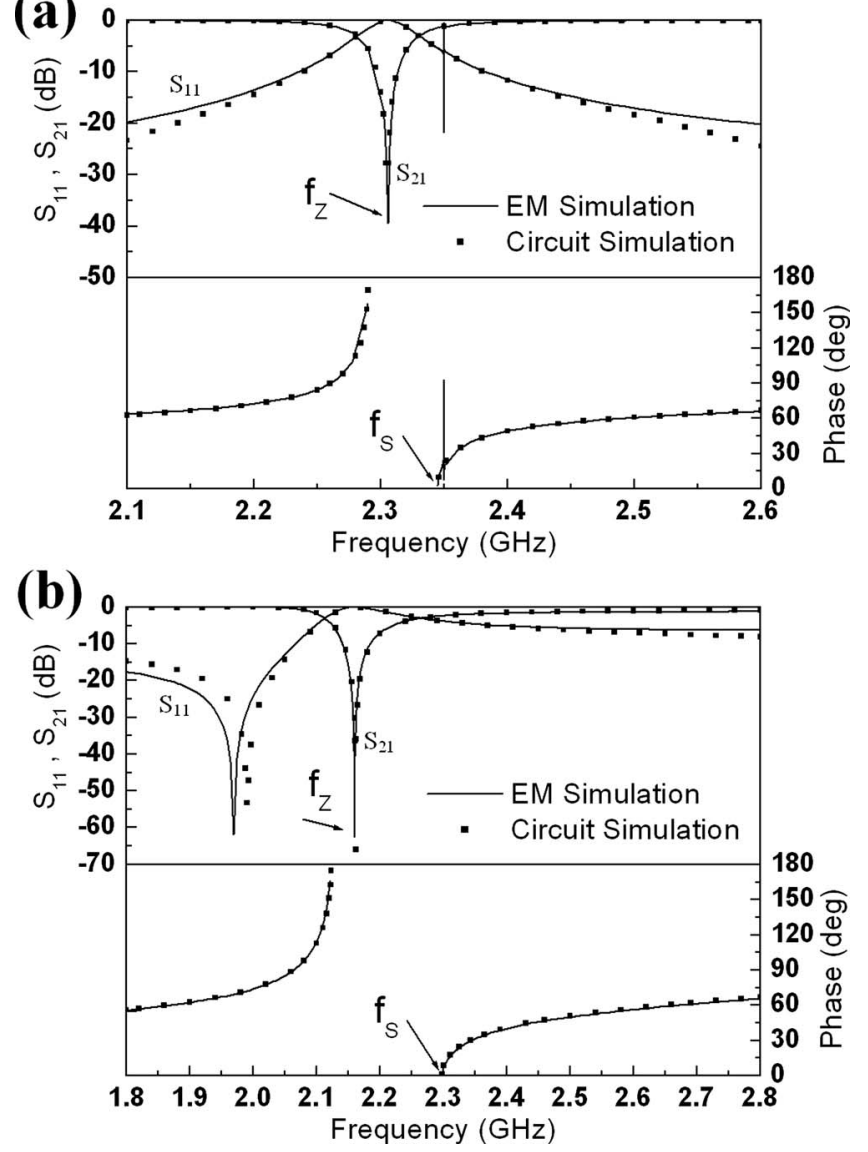

FIG. 6. Simulated reflection, $S_{11}$, and transmission, $S_{21}$, coefficients, and simulated dispersion relation for negative-permeability cells based on microstrip (a) and CPW (b) structures. The layouts are represented in Fig. 1 removing the shunt elements. The considered substrate and the relevant dimensions are the same as those in Figs. 4 and 5.

obtained from the electromagnetic simulation together with the electrical response corresponding to the circuit obtained from the parameter extraction. Both responses are identical, as what occurs in Fig. 5 with the SRR with a length $\lambda_{g} / 10$. It should be taken into account that for these resonators with two metal layers, three metal layers are necessary: two for the resonator and one more for the coplanar line. The circuit parameters are shown in Table II. With these results, it is demonstrated that the parameter extraction method is suitable for any subwavelength resonator.

Thus, we can conclude that the circuit model shown in Fig. 2 appropriately describes the behavior of left handed lines loaded with magnetically coupled resonators. The proposed parameter extraction method is simple and useful for the characterization of resonant type metamaterial transmission lines.

\section{DISCUSSION}

The accurate determination of the parameters of the isolated particles (that is, without the presence of the CPW metal level) from the simulation of the structure proposed in Fig. 1(b) and applying the parameter extraction proposed in Sec. II is not possible since, to this end, the value of the mutual coupling $M$ is required, and the assumption $M=L f(f$ being the fraction of the slots occupied by the resonators) is 
(a) BC-NB-SRR

(b) BC-SR(2)

(c) BC-SR(4)
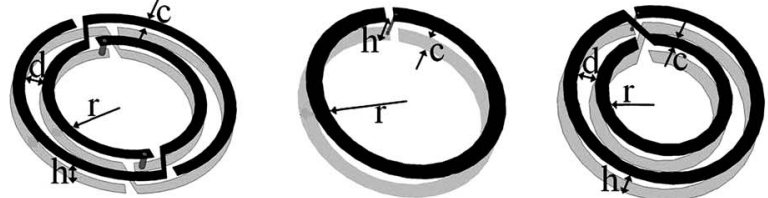

(d)

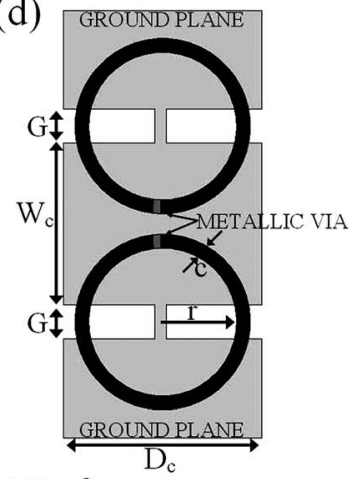

(e)
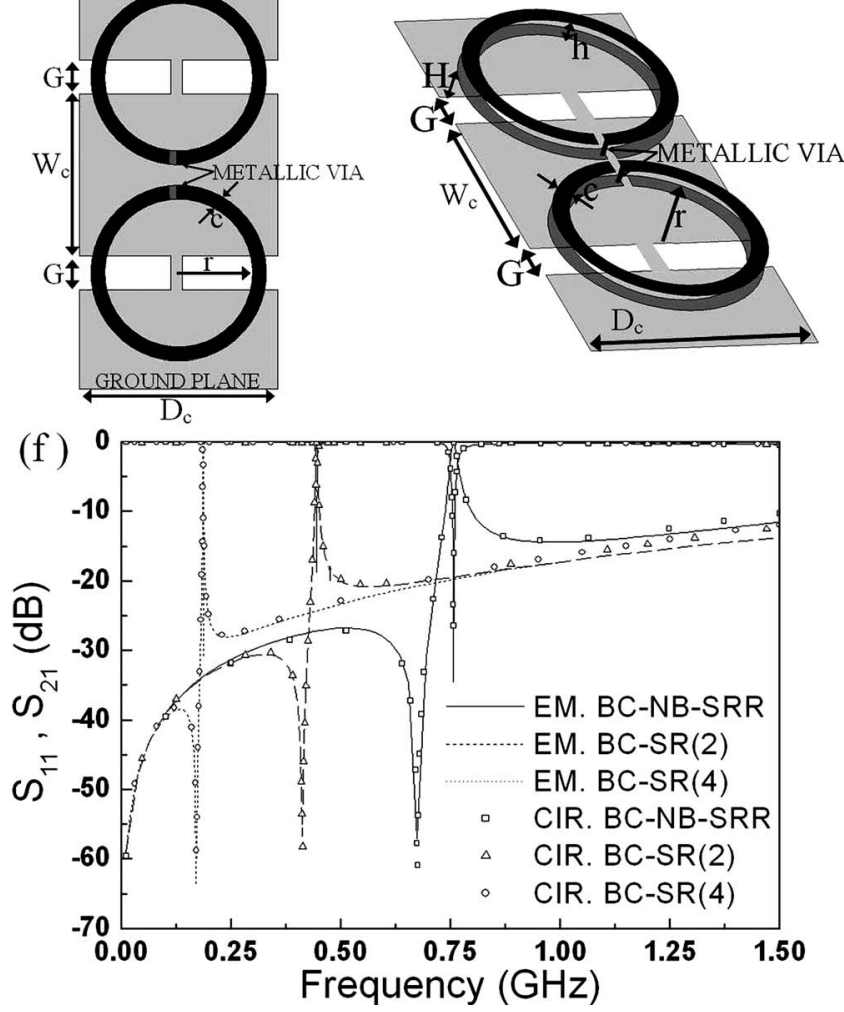

FIG. 7. Topologies of the different resonators considered: BC-NB-SRR (a), BC-SR(2) (b), and BC-SR(4) (c). Layout of a three metal layer configuration of a CPW loaded with a pair of metamaterial resonators and shunt strips: top (d) and three dimensional (e) views. Frequency responses (f) for three different resonators: BC-NB-SRR, BC-SR(2), and BC-SR(4) coupled to CPW structure with shunt strips. The considered substrate is Rogers RO3010 with dielectric constant $\varepsilon_{r}=10.2$. The relevant dimensions for the resonators are ring width $c=0.6 \mathrm{~mm}$; distance between the rings, $d=0.2 \mathrm{~mm}$; and internal radius $r=2.4 \mathrm{~mm}$ for the $\mathrm{BC}-\mathrm{NB}-\mathrm{SRR}$ and the $\mathrm{BC}-\mathrm{SR}(4)$ and $r=3.2 \mathrm{~mm}$ for the BC-SR(2). The substrate thickness between the resonator metallic layers is $h=0.635 \mathrm{~mm}$ for the BC-NB-SRR and $h=0.127 \mathrm{~mm}$ for the BC$\mathrm{SR}(2)$ and the BC-SR(4). For the CPW structure the central strip width is $W_{c}=7 \mathrm{~mm}$, the width of the slots is $G=1.35 \mathrm{~mm}$, and the length is $D_{c}$ $=8.6 \mathrm{~mm}$. The shunt strip width is $w_{s}=0.4 \mathrm{~mm}$; the substrate thickness between the CPW and the resonators is $H=0.635 \mathrm{~mm}$ for the BC-NB-SRR and $H=1.143 \mathrm{~mm}$ for the BC-SR(2) and the BC-SR(4). The results of the electrical simulation with extracted parameters are depicted using symbols.

only an approximation. Indeed, this assumption is valid as long as the following conditions are fulfilled: (i) the resonant elements can be described through a quasistatic analysis and (ii) the magnetic flux lines generated by the line in the region of the resonators penetrate the whole resonator area. As long as the resonators are electrically small, the first condition is reasonable, but it is not likely that the second condition applies for relatively thick dielectric layers (as those of many low loss commercial microwave substrates).

Let us assume however that $M$ is known. In that case, the parameters of the model in Fig. 3 can be determined
TABLE II. Extracted parameters for the circuit shown in Fig. 2 with a CPW structure using different pairs of coupled resonators.

\begin{tabular}{lccccc}
\hline \hline & $\begin{array}{c}C \\
(\mathrm{pF})\end{array}$ & $\begin{array}{c}L^{\prime} \\
(\mathrm{nH})\end{array}$ & $\begin{array}{c}L_{p}^{\prime} \\
(\mathrm{nH})\end{array}$ & $\begin{array}{c}C_{s}^{\prime} \\
(\mathrm{pF})\end{array}$ & $\begin{array}{c}L_{s}^{\prime} \\
(\mathrm{nH})\end{array}$ \\
\hline BC-NB-SRR & 3.37 & 4.01 & 2.08 & 25.80 & 2.16 \\
BC-SR(2) & 2.00 & 4.71 & 2.21 & 102.03 & 1.46 \\
BC-SR(4) & 2.20 & 4.39 & 2.20 & 545.11 & 1.59 \\
\hline \hline
\end{tabular}

through transformations (5)-(8). However, notice that $L_{s}$ and $C_{s}$ are the resonator parameters influenced by the presence of the metallic CPW structure on the opposite substrate side, which are different from the parameters of the isolated particles, $L_{I}$ and $C_{I}$. $L_{I}$ can be inferred from $L_{s}$ by means of the method of images according to

$$
L_{I}=L_{s}+M^{\prime},
$$

where $M^{\prime}$ is the mutual coupling between the resonator and its image, and it has been considered that the effect of the slots, in the case of the CPW, is negligible. $M^{\prime}$ is obtained by means of the well known Neumann's formula, where infinitely thin metal loops have been considered, with the radius corresponding to the average radius of the used resonator.

The determination of $C_{I}$ from $C_{s}$ is more complicated, but for relatively thick substrates (as compared to the distance between concentric rings, $c$ ) it is expected that $C_{I}$ $\approx C_{s}$. A key point is if there exists a range of substrate thicknesses for which $C_{I} \approx C_{s}$ and the assumption $M=L f$ is valid. To this end, we have calculated for four different particles the value of mutual coupling $M_{t}$ that makes $C_{I} \approx C_{s}\left(C_{I}\right.$ and $L_{I}$ can be calculated analytically for the considered particles), and we have calculated the ratio $M_{t} / M$ (normalized coupling). Notice that the normalized coupling coincides with $f_{t} / f$, where $f_{t}=M_{t} / L$. We may call $f_{t} / f$ the normalized slot fraction. Such normalized slot fraction is depicted in Fig. 8 for different substrate thicknesses. The considered particles

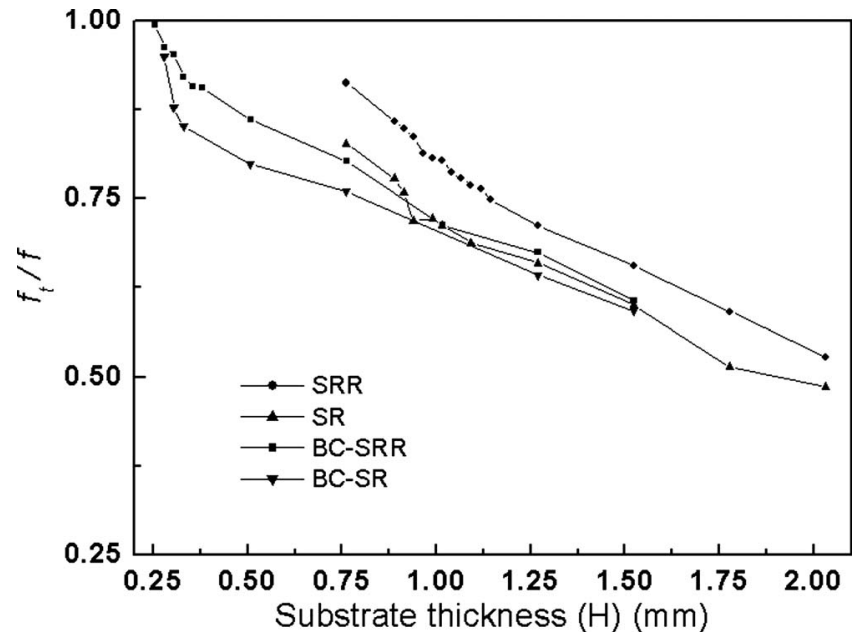

FIG. 8. Normalized slot fractions $\left(f_{t} / f\right)$ that make $C_{I}=C_{s}$ for four different resonant particles and different substrate thicknesses $H$. The relevant dimensions for the resonators are ring width $c=0.6 \mathrm{~mm}$, distance $d=0.2 \mathrm{~mm}$ between the rings, and internal radius $r=2.4 \mathrm{~mm}$ for the SRR and the SR; and $c=0.6 \mathrm{~mm}, r=3.2 \mathrm{~mm}$, and substrate thickness $h=0.127 \mathrm{~mm}$ between layers for the BC-SRR and the BC-SR(2). 
TABLE III. Extracted parameters for the SRR-loaded microstrip and CPW unit cells with and without shunt strips (second and third columns) and isolated parameters (fifth and sixth columns). Modified mutual inductances (first column) and mutual couplings between the resonator and its image (fourth column).

\begin{tabular}{lcccccc}
\hline \hline Structure & $\begin{array}{c}M_{t} \\
(\mathrm{nH})\end{array}$ & $\begin{array}{c}C_{s}, C_{I}, C_{I \mathrm{mod}} \\
(\mathrm{pF})\end{array}$ & $\begin{array}{c}L_{s} \\
(\mathrm{nH})\end{array}$ & $\begin{array}{c}M^{\prime} \\
(\mathrm{nH})\end{array}$ & $\begin{array}{c}L_{I}=L_{s}+M^{\prime} \\
(\mathrm{nH})\end{array}$ & $\begin{array}{c}L_{I \mathrm{mod}} \\
(\mathrm{nH})\end{array}$ \\
\hline \multicolumn{7}{c}{ With the shunt metallic elements } \\
Microstrip & 0.70 & 0.50 & 9.58 & 2.1 & 11.68 & 12.55 \\
CPW & 1.13 & 0.50 & 10.43 & 2.1 & 12.53 & 12.55 \\
\multicolumn{7}{c}{} \\
Microstrip & 0.71 & 0.50 & 9.69 & 2.1 & 11.79 & 12.55 \\
CPW & 1.17 & 0.50 & 10.41 & 2.1 & 12.51 & 12.55 \\
\hline \hline
\end{tabular}

have the same external radius. Such particles are SRRs, SRs, BC-SRR, and BC-SR(2). As can be seen, the normalized slot fraction decreases with substrate thickness and it is the same for all the considered resonators (that corresponding to the SRR is slightly above the others for unknown reasons). That is, as the thickness increases, the fraction of the magnetic flux lines generated by the line that penetrates the resonator area decreases and $M_{t}<M\left(f_{t}<f\right)$. Except for two metal layer resonators with significant intermetallic distance, the normalized slot fraction is expected to depend essentially on the substrate thickness, as Fig. 8 demonstrates. However, notice that as substrate thickness is reduced, rather than a saturation of the normalized slot fraction (that is, $f_{t} / f \rightarrow 1$ ), this normalized fraction rapidly increases. This occurs for substrate thicknesses below approximately $0.50 \mathrm{~mm}$, where the assumption that $C_{I} \approx C_{s}$ fails, so that neither $M_{t}$ nor $f_{t}$ have a physical meaning. Thus, the presented results show that there is not a region where the actual coupling $M_{t}$ coincides with $M=L f$ and, simultaneously, $C_{I} \approx C_{s}$. This precludes the accurate determination of the parameters of the isolated particles $C_{I}$ and $L_{I}$. However, we can estimate these parameters by considering a test structure consisting on a CPW with thickness above $1.5 \mathrm{~mm}$ and use the normalized slot fraction derived from Fig. 8 (the average value). With these values, the mutual coupling $M_{t}$ can be inferred and by introducing it in Eqs. (5)-(8), we can infer $L_{s}$ and $C_{s}$ and finally the parameters of the isolated particle, given by Eq. (14) and $C_{I}$ $=C_{s}$.

To confirm the coherence between the extracted parameters (circuit in Fig. 2) and those of the isolated particles, we have considered the CPW and microstrip structures in Fig. 1, where we have extracted the parameters and we have calculated the mutual coupling $M_{t}$ necessary to obtain $C_{I}=C_{s}$ (the substrate thickness is large enough). Actually this has been done also by removing the shunt connected inductances (strips or vias). The values of $M_{t}, C_{s}, L_{s}, M^{\prime}, C_{I}$, and $L_{I}$ are given in Table III, together with the analytical values of $C_{I}$ and $L_{I}$ (obtained from reported models ${ }^{8,19,30}$ and termed as $C_{I \text { mod }}$ and $L_{I \text { mod }}$ in Table III). As can be seen the analytical $\left(L_{I \mathrm{mod}}\right)$ and calculated $\left(L_{I}\right)$ resonator inductances are in reasonable agreement (obviously $C_{s}=C_{I}$ since we have forced this). To further support the previous analysis, we have considered the four resonators in Fig. 8, each one loading a
TABLE IV. Extracted parameters for the SRR-, SR-, BC-SRR-, and BC-SRloaded CPW unit cells without shunt strips for two substrate thicknesses.

\begin{tabular}{|c|c|c|c|c|c|c|c|c|}
\hline & $\begin{array}{c}C \\
(\mathrm{pF})\end{array}$ & $\begin{array}{c}L \\
(\mathrm{nH})\end{array}$ & $\begin{array}{c}M \\
(\mathrm{nH})\end{array}$ & $\begin{array}{c}C_{s}, \underset{(\mathrm{pF})}{C_{I}, C_{I \bmod }} \\
\end{array}$ & $\begin{array}{c}L_{s} \\
(\mathrm{nH})\end{array}$ & $\begin{array}{c}M^{\prime} \\
(\mathrm{nH})\end{array}$ & $\begin{array}{c}L_{I}=L_{S}+M^{\prime} \\
(\mathrm{nH})\end{array}$ & $\begin{array}{l}L_{\text {Imod }} \\
(\mathrm{nH})\end{array}$ \\
\hline \multicolumn{9}{|c|}{ Substrate thickness $H=1.27 \mathrm{~mm}$} \\
\hline SRR & 2.38 & 2.27 & 1.17 & 0.50 & 10.41 & 2.1 & 12.51 & 12.55 \\
\hline SR & 2.03 & 2.49 & 1.19 & 2.00 & 9.96 & 2.1 & 12.06 & 12.55 \\
\hline BC-SRR & 2.00 & 2.71 & 1.38 & 2.62 & 13.33 & 2.5 & 15.83 & 14.70 \\
\hline BC-SR & 1.95 & 2.68 & 1.36 & 10.48 & 13.14 & 2.5 & 15.64 & 14.70 \\
\hline \multicolumn{9}{|c|}{ Substrate thickness $H=1.78 \mathrm{~mm}$} \\
\hline SRR & 2.05 & 2.32 & 1.00 & 0.50 & 10.53 & 1.3 & 11.83 & 12.55 \\
\hline SR & 1.80 & 2.76 & 1.03 & 2.00 & 9.83 & 1.3 & 11.13 & 12.55 \\
\hline BC-SRR & 1.85 & 2.51 & 1.15 & 2.62 & 13.49 & 1.7 & 15.19 & 14.70 \\
\hline BC-SR & 1.78 & 3.50 & 1.34 & 10.48 & 13.50 & 1.7 & 15.20 & 14.70 \\
\hline
\end{tabular}

CPW structure without strips, and we have obtained the same parameters as in Table III. We have done this for two different thicknesses. The results are shown in Table IV. Again, there is reasonable agreement between $L_{I \bmod }$ and $L_{I}$. (Indeed, taking into account that the width of the resonators is relatively wide $-c=0.6 \mathrm{~mm}$ - some deviation between the calculation and the analytical model can be expected.)

The reported parameter extraction technique for the circuit in Fig. 2 provides results that are coherent with the parameters of the resonators analytically obtained. The parameter extraction technique is very valuable and valid for complex resonators which cannot be easily modeled, such as the ones presented in Ref. 29. We would like to mention that the resonator parameters can also be extracted from experimental data. However, losses may obscure the results and the comparison to the analytical models. For this reason this study has been restricted to frequency responses inferred from electromagnetic simulation. Nevertheless, it is well known that the electromagnetic software that has been used (AGILENT MOMENTUM) predicts the behavior of the resonators with good accuracy, as has been previously demonstrated, thus supporting the validity of this study.

\section{CONCLUSIONS}

In conclusion, we have proposed a technique for the determination of resonator parameters when they are coupled to a planar transmission line. The technique has been applied to several resonant particles with different electrical lengths and different structures in microstrip and CPW configurations. We have discussed the possibility of obtaining the parameters of the isolated particles from the extracted parameters, and we have concluded that this cannot be done accurately, although we can obtain a reasonable estimation. Nevertheless, we have corroborated that the extracted parameters are coherent with the parameters of the isolated particles, and this has been done for different host lines, particles, and substrate thicknesses.

\section{ACKNOWLEDGMENTS}

This work was supported by Spain-MEC (Project Contract No. TEC2007-68013-C02 METAINNOVA). Thanks are 
also given to the European Union for funding the Network of Excellence (NoE) METAMORPHOSE, and to the Catalan government (CIDEM) for funding CIMITEC and for giving support to the UAB team through Project No. 2005SGR0624. MEC gave an FPU grant to M.G. (Ref. No. AP20054523).

${ }^{1}$ F. Martín, F. Falcone, J. Bonache, R. Marqués, and M. Sorolla, Appl. Phys. Lett. 83, 4652 (2003).

${ }^{2}$ J. B. Pendry, A. J. Holden, D. J. Robbins, and W. J. Stewart, IEEE Trans. Microwave Theory Tech. 47, 2075 (1999)

${ }^{3}$ F. Falcone, T. Lopetegi, M. A. G. Laso, J. D. Baena, J. Bonache, R Marqués, F. Martín, and M. Sorolla, Phys. Rev. Lett. 93, 197401 (2004).

${ }^{4}$ F. Falcone, T. Lopetegi, J. D. Baena, R. Marqués, F. Martín, and M. Sorolla, IEEE Microw. Wirel. Compon. Lett. 14, 280 (2004).

${ }^{5}$ R. Marqués, F. Martín, and M. Sorolla, Metamaterials with Negative Parameters: Theory, Design and Microwave Applications (Wiley, New Jersey, 2007).

${ }^{6}$ G. V. Eleftheriades and K. G. Balmain, Negative Refraction Metamaterials: Fundamental Principles and Applications (Wiley, New Jersey, 2005).

${ }^{7} \mathrm{C}$. Caloz and T. Itoh, Electromagnetic Metamaterials: Transmission Line Theory and Microwave Applications (Wiley, New Jersey, 2006).

${ }^{8}$ R. Marqués, F. Medina, and R. Rafii-El-Idrissi, Phys. Rev. B 65, 144440 (2002).

${ }^{9}$ J. García-García, F. Martín, J. D. Baena, and R. Maqués, J. Appl. Phys. 98, 033103 (2005)

${ }^{10}$ I. H. Lin, M. De Vincentis, C. Caloz, and T. Itoh, IEEE Trans. Microwave Theory Tech. 52, 1142 (2004).

${ }^{11}$ M. A. Antoniades and G. V. Eleftheriades, IEEE Microw. Wirel. Compon. Lett. 15, 808 (2005).

${ }^{12}$ H. Okabe, C. Caloz, and T. Itoh, IEEE Trans. Microwave Theory Tech. 52 , 798 (2004).

${ }^{13}$ G. Sisó, J. Bonache, M. Gil, J. García-García, and F. Martín, IEEE MTT-S Int. Microwave Symp. Dig. 25 (2007)
${ }^{14}$ I. B. Vendik, O. G. Vendik, D. V. Kholodnyak, E. V. Serebryakova, and P. V. Kapitanova, Proceedings of the European Microwave Association, Vol. 2, p. 30 (2006)

${ }^{15}$ M. Gil, J. Bonache, J. García-García, and F. Martín, IEEE MTT-S Int. Microwave Symp. Dig. 1419 (2007).

${ }^{16}$ M. Gil, J. Bonache, I. Gil, J. García-García, and F. Martín, Int. J. Numer. Model. 19, 87 (2006).

${ }^{17}$ J. Bonache, M. Gil, I. Gil, J. García-García, and F. Martín, IEEE Microw. Wirel. Compon. Lett. 16, 543 (2006).

${ }^{18}$ M. Gil, J. Bonache, I. Gil, J. García-García, and F. Martín, IET Proc. Microwaves, Antennas Propag. 1, 73 (2007).

${ }^{19}$ R. Marqués, F. Mesa, J. Martel, and F. Medina, IEEE Trans. Antennas Propag. 51, 2572 (2003).

${ }^{20}$ F. Aznar, J. García-García, J. Bonache, M. Gil, and F. Martín, Electron. Lett. 43, 530 (2007).

${ }^{21}$ I. Gil, J. Bonache, M. Gil, J. García-García, F. Martín, and R. Marqués, J. Appl. Phys. 100, 074908 (2006).

${ }^{22}$ E. Shamonina, V. A. Kalinin, K. H. Ringhofer, and L. Solymar, J. Appl. Phys. 92, 6252 (2002).

${ }^{23}$ M. C. K. Wiltshire, E. Shamonina, I. R. Young, and L. Solymar, Electron. Lett. 39, 215 (2003).

${ }^{24}$ M. J. Freire, R. Marqués, F. Medina, M. A. G. Laso, and F. Martín, Appl. Phys. Lett. 85, 4439 (2004)

${ }^{25}$ R. R. A. Syms, E. Shamonina, and L. Solymar, J. Appl. Phys. 97, 064909 (2005).

${ }^{26}$ M. Beruete, M. J. Freire, R. Marqués, F. Falcone, and J. D. Baena, Appl. Phys. Lett. 88, 083503 (2006).

${ }^{27}$ I. Gil, J. Bonache, J. García-García, and F. Martín, IEEE Trans. Microwave Theory Tech. 54, 2665 (2006).

${ }^{28}$ F. Aznar, J. Bonache, and F. Martín, Appl. Phys. Lett. 92, 043512 (2008).

${ }^{29}$ F. Aznar, J. García-García, M. Gil, J. Bonache, and F. Martín, Microw. Opt. Technol. Lett. 50, 1263 (2008).

${ }^{30}$ J. D. Baena, J. Bonache, F. Martín, R. M. Sillero, F. Falcone, T. Lopetegi, M. A. G. Laso, J. García-García, I. Gil, M. F. Portillo, and M. Sorolla, IEEE Trans. Microwave Theory Tech. 53, 1451 (2005). 